\title{
Comparison of treatments for hepatocellular carcinoma patients with portal vein thrombosis: a systematic review and network meta-analysis
}

\author{
Jianchen Luo ${ }^{1} \wedge$, Liangliang $\mathrm{Xu}^{2} \wedge$, Lian $\mathrm{Li}^{1 \wedge}$, Jingfu Zhang ${ }^{1} \wedge$, Ming Zhang ${ }^{2} \wedge$, Mingqing $\mathrm{Xu}^{2} \wedge$ \\ ${ }^{1}$ Department of General Surgery, West China Hospital, Sichuan University, Chengdu, China; ${ }^{2}$ Liver Surgery and Liver Transplantation Center, West \\ China Hospital, Sichuan University, Chengdu, China \\ Contributions: (I) Conception and design: J Luo; (II) Administrative support: M Xu; (III) Provision of study materials or patients: J Luo, M Zhang; \\ (IV) Collection and assembly of data: J Luo, L Li; (V) Data analysis and interpretation: J Luo, L Xu; (VI) Manuscript writing: All authors; (VII) Final \\ approval of manuscript: All authors. \\ Correspondence to: Mingqing Xu. Liver Surgery and Liver Transplantation Center, West China Hospital, Sichuan University, No. 37 Guo Xue Xiang, \\ Chengdu 610041, China. Email: xumingqing0018@163.com.
}

\begin{abstract}
Background: Sorafenib, hepatectomy, and transarterial chemoembolization (TACE) are the recommended treatment for portal vein tumor thrombosis (PVTT) patients. Therefore, the aim of the present study was to conduct a multi-treatment meta-analysis. The aim of the present study was to analyze the survival benefit of different treatments options on PVTT patients.

Methods: We systematically analyzed 12 randomized controlled trials (4,265 participants) from 2012 to 2019, which compared any of the following treatment options on PVTT patients: TACE, sorafenib, hepatectomy, sorafenib + TACE, hepatectomy + TACE, and sorafenib + hepatectomy. The main outcome was the 1-year survival rate of patients.

Results: The results of the rank probability of effectiveness showed that sorafenib + TACE was more likely to be the most effective treatment, sorafenib + TACE group was ranged rank 1 when compared with the others [hepatectomy group: odds ratio (OR): 0.79, 95\% confidence interval (CI): 0.03-18.26; hepatectomy + TACE group: OR: 0.51, 95\% CI: 0.01-13.59; sorafenib group: OR: 0.14, 95\% CI 0.01-2.29, sorafenib + hepatectomy group: OR: 0.15, 95\% CI: 0.00-24.88; and TACE group: OR: 0.51, 95\% CI: 0.02-9.88]. The second most effect treatment option was hepatectomy alone.

Discussion: Sorafenib + TACE is more likely to be the most effective treatment option, while hepatectomy alone is the second effective treatment option.
\end{abstract}

Keywords: Hepatocellular carcinoma (HCC); portal vein tumor thrombosis (PVTT); Bayesian framework

Submitted Jul 21, 2021. Accepted for publication Aug 26, 2021.

doi: 10.21037/atm-21-3937

View this article at: https://dx.doi.org/10.21037/atm-21-3937

\section{Introduction}

Hepatocellular carcinoma (HCC) is the fifth most common cancer globally, and is one of the most prevalent causes of cancer-related death (1). HCC is likely to invade the portal vein system during the tumor growth (2). Poon et al. claimed that the incidence of portal vein tumor thrombosis (PVTT) in patients with liver cancer is as high as $43 \%$,

^ ORCID: Jianchen Luo, 0000-0002-1352-4140; Liangliang Xu, 0000-0002-3900-9972; Lian Li, 0000-0002-8517-4661; Jingfu Zhang, 0000-0002-5069-3496; Ming Zhang, 0000-0002-8276-691X; Mingqing Xu, 0000-0002-8556-0802. 
while $64.7 \%$ of patients with HCC may get portal vein invasion according to the autopsy report (3). However, patients with PVTT are often diagnosed at an advanced stage and have a short survival time. The Barcelona Clinic Liver Cancer/American Association for the Study of Liver Diseases classifies these patients as C stage (4). If PVTT patients are not treated, their median survival time is only 2.7 months (5-7). Sorafenib is recommended as the only treatment option, but the survival of PVTT patients is still unfavorable. Treatment methods, such as surgery and transarterial chemoembolization (TACE), are also implemented. According to a previously published study, the median survival time of PVTT patients can be as short as 10.7 months (8).

With the rapid evolvement of surgical techniques and equipment as well as the rise of adjuvant treatment measures, perioperative management is becoming gradually standardized. PVTT patients get more treatment options (9). Based on new research advances, Japanese scientists have claimed that hepatectomy can prolong the survival time of PVTT patients compared with those who do not undergo surgery (2.45 vs. 1.57 years, respectively; $\mathrm{P}<0.001)$ (4). Reports of the therapeutic effects of Sorafenib also tend to be favorable (8). Therefore, hepatectomy and sorafenib may play a more important role in the treatment of PVTT patients.

Evidence also suggests that TACE can be more effective compared with hepatic resection, and the survival of HCC patients with PVTT can be further prolonged. (4,10-12). TACE is considered to be an update for traditional treatments, because TACE was thought might lead to post-operational liver dysfunction in these PVTT patients (13). However, in recent studies, the process of tumor thrombus formation has been described from the perspective of disease development, and TACE has been indicated to be an alternative option for PVTT patients. This view was also supported in the 2010 International Hepatobiliary Association Expert Consensus Statement, which recommends TACE as a standard treatment for PVTT patients $(14,15)$. In addition, the treatment effect of sorafenib chemotherapy compared with TACE and hepatectomy is still controversial. Recent studies had compared hepatectomy, TACE, and sorafenib as separate groups in pairs $(16,17)$. Whereas in clinical practice, one patient may receive two or more treatments at the same time or in succession. Whether a combination of the multiple treatments above is better than a monotherapy remains to be proved.
We conducted a Bayesian framework to compare the efficacy of sorafenib, TACE and hepatectomy alone or in combination. Data from previously reported studies were collected and reviewed (using both direct and indirect comparisons). The aim of the present study was to analyze the survival benefit of six different treatments options on PVTT patients. We present the following article in accordance with the PRISMA reporting checklist (available at https://dx.doi.org/10.21037/atm-21-3937).

\section{Methods}

\section{Literature search}

Prior to study commencement, a study protocol was drafted, which is approved by Liver Surgery and Liver Transplantation Center, West China Hospital of Sichuan University. The overall dataset will be in the public domain upon publication of this paper.

Literature retrieval was independently done by two authors. To obtain potentially eligible studies, we searched PubMed, Embase, Cochrane Library, and Web of Science. The search strategy was as follows: (hepatectomy OR hepatic resection OR liver resection) OR (transcatheter arterial chemoembolization OR TACE) OR (chemotherapy OR TACE OR sorafenib) AND (portal vein invasion OR portal vein tumor thrombosis OR PVTT) AND (HCC OR hepatocellular carcinoma OR liver cancer). The reference lists of relative reviews and meta-analyses were also manually screened to identify more studies.

\section{Study selection}

We screened the references cited in the retrieved articles. The selected studies compared any 2 of the 6 treatments (TACE, sorafenib, hepatectomy, sorafenib + TACE, hepatectomy + TACE, and sorafenib + hepatectomy). Each study was reviewed by two authors and the results of each study were recorded.

The screening criteria were as follows: (I) the study was a prospective or retrospective study comparing PVTT patients treated with those 6 treatments; (II) the results of the study showed a definite survival 1-year survival rate; and (III) the study reported sufficient data to estimate the hazard ratio (HR) and $95 \%$ confidence interval (CI).

The exclusion criteria were as follows: (I) no HCC; (II) no comparative studies; (III) comparison of recurrent or ruptured HCC or mixed malignances; (IV) studies without 
valuable data or data acquired through animal experiments; and $(\mathrm{V})$ reviews, letters, case reports, and expert opinions.

\section{Study synthesis, data extraction and analysis}

Two reviewers independently extracted the data and assessed the validity in the identified studies according to a predetermined procedure, including basic information, such as the article title, the first author's name, and the year of publication; the baseline characteristics observed in the studies, including age, sex, hepatitis B virus, cirrhosis, and tumor characteristics; and specific information on the treatment option (intervention), such as the duration of the intervention, the collection time of intervention cases, the length of follow up, different types of PVTT, and outcomes (1-year survival rate). Treatment effectiveness was based on the improvement of clinical symptoms, and then the effective rate was calculated. When there was any doubt regarding the data extraction, discussions took place with the third author to reach consensus, and the authors of the eligible studies were contacted if necessary. The data were collected and descriptively summarized to enable the further examination of variations in the research characteristics and results. This process helped to confirm the similarities between studies and the applicability of further synthesis methods (18).

\section{Sorafenib administration and PVTT}

In all of the included articles, approximately $400 \mathrm{mg}$ sorafenib was used for treatment (Provided by Bayer HealthCare AG, $200 \mathrm{mg} /$ pill), and was orally given twice daily. When grade 3 or 4 adverse events (e.g., skin, hematological, and gastrointestinal toxicities or organ dysfunction, as defined by the National Cancer Institute Common Terminology Criteria for Adverse Events) occurred (19), the oral dose was reduced to $200 \mathrm{mg}$ per day. The dose was adjusted if there were symptoms of adverse reactions.

PVTT was classified according to four types, as described by Shi et al. Type I PVTT was defined as tumor thrombi involving the segmental branches of the portal vein or above. Type II PVTT was defined as tumor thrombi extending to involve the right/left portal vein. Type III PVTT was defined as thrombi involving the main portal vein. Type IV PVTT was defined as thrombi involving the superior mesenteric vein (20).

\section{Statistical analysis}

All the studies were screened following the Preferred Reporting Items for Systematic Reviews and Meta-Analyses (PRISMA) extension statement for reporting systematic reviews incorporating network meta-analyses of healthcare interventions. A Bayesian hierarchical random-effects model was used to compare the outcome variables using relative ORs and CS calculated with RStudio version 4.0.2 and ADDIS. Trials with 3 or more arms were split into 2 -arm trials if they could be combined. We used visual inspection of the forest plots to investigate the possibility of statistical heterogeneity, and the $\mathrm{I}^{2}$ statistic. Inconsistencies between pairwise effect estimates and between direct and indirect effect estimates were assessed with the $\mathrm{I}^{2}$ statistic, with values below $40 \%$ representing moderate inconsistency and values over $75 \%$ representing severe inconsistency. In the Markov Chain Monte Carlo chain (MCMC chain), convergence and lack of autocorrelation were checked and confirmed after 100,000 iterations. RevMan software version 5.3 was used to evaluate the risk of bias in the eligible studies.

\section{Results}

\section{Characteristics of included studies}

In total, 819 studies were retrieved in PubMed, Embase, Cochrane Library, and Web of Science. After the removal of duplicates, 529 unique records remained; 316 articles were removed by title screening and 188 articles were removed by abstract screening. The full text of the remaining records was then examined, and 12 eligible randomized controlled trials were identified for the present network metaanalysis. A total of 4,265 cases were included in our study (Figure 1) (12,21-31). Of these, 477 patients were in the sorafenib group, 1,633 were in the TACE group, 1,532 were in the hepatectomy group, 20 were in the sorafenib + hepatectomy group, 135 were in the sorafenib + TACE group, and 285 were in the hepatectomy + TACE group. All patients had historically confirmed PVTT before these treatments. Rates of survival at postoperative follow up were reported in all the literature. The characteristics of the included studies and of the patients are shown in Table 1. Methods for handling missing data and intentionto-treat analysis were not adequately described in most of the studies. 


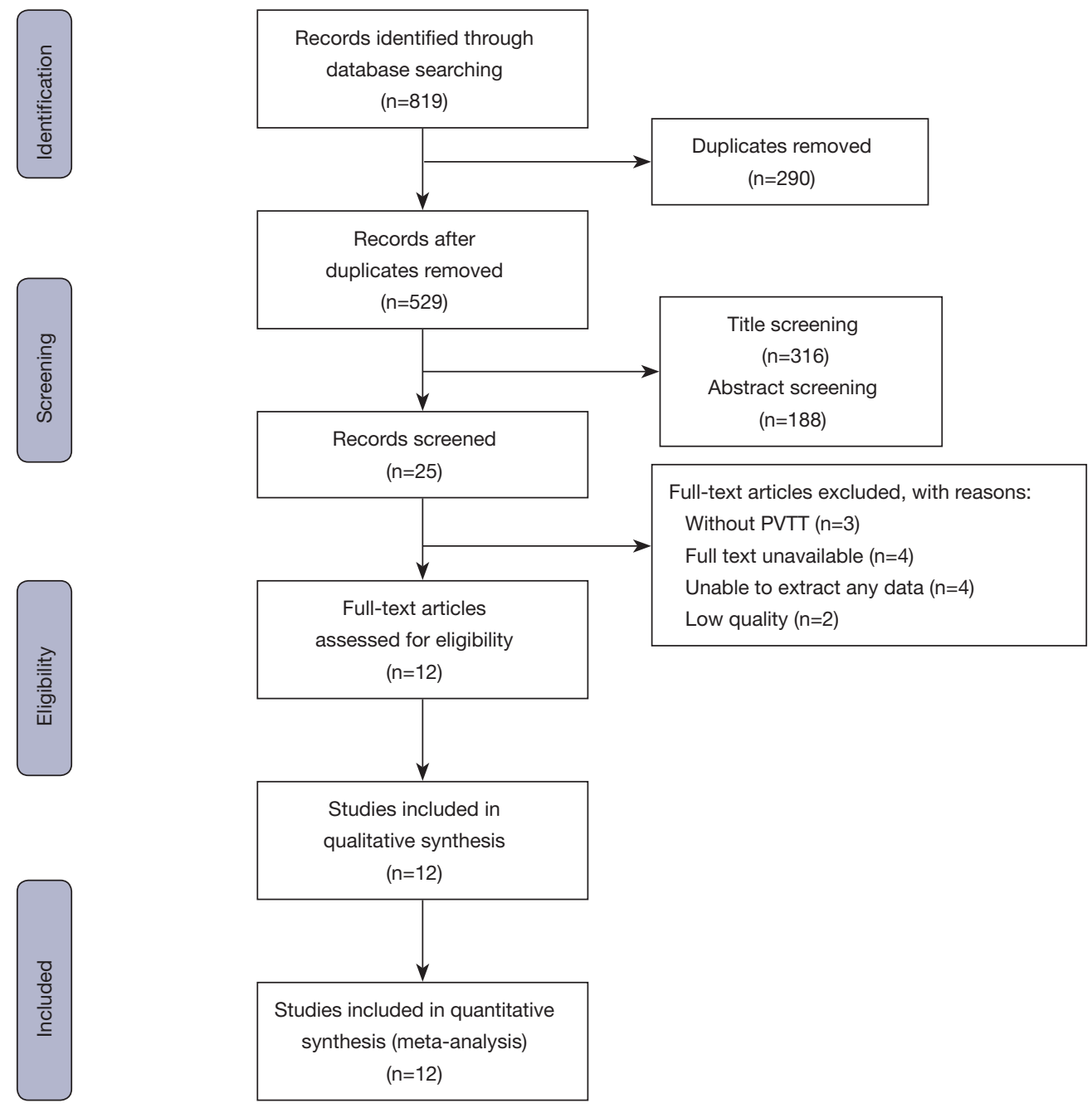

Figure 1 Study selection. PVTT, portal vein tumor thrombosis.

\section{Risk of bias}

There was a low risk of bias in random-sequence generation in all of the studies $(12,21-31)$. No publication bias was found in the analysis. All of the studies had a low risk of bias in incomplete outcome data. Only 2 studies had a high risk of bias in the blinding procedure of participants and personnel (Figure S1) $(21,25)$.

\section{Network structure and heterogeneity}

A total of 12 articles were included, forming 31 comparisons in the analysis. The network structure of the eligible studies is shown in Figure 2. Non-significant heterogeneity among trials was viewed as acceptable $\left(\mathrm{I}^{2}<75 \%\right)$. Each node represented a treatment. Directly compared treatments were linked with a line, and the thickness of these lines reflected the number of trials that were assessed in the comparison.

\section{Results of the MCMC chain and density plot}

The result of the trace plots of the MCMC chain were provided to assess the convergence of the analysis, the result showed that the convergence could be confirmed accurately after 100,000 iterations (32). The plots are shown in Figure 3.

The density plot showed that all the bandwidth values were close to 0 . That meant that the convergence could be confirmed accurately after 100,000 iterations $(33,34)$. The density plot is shown in Figure 3. 


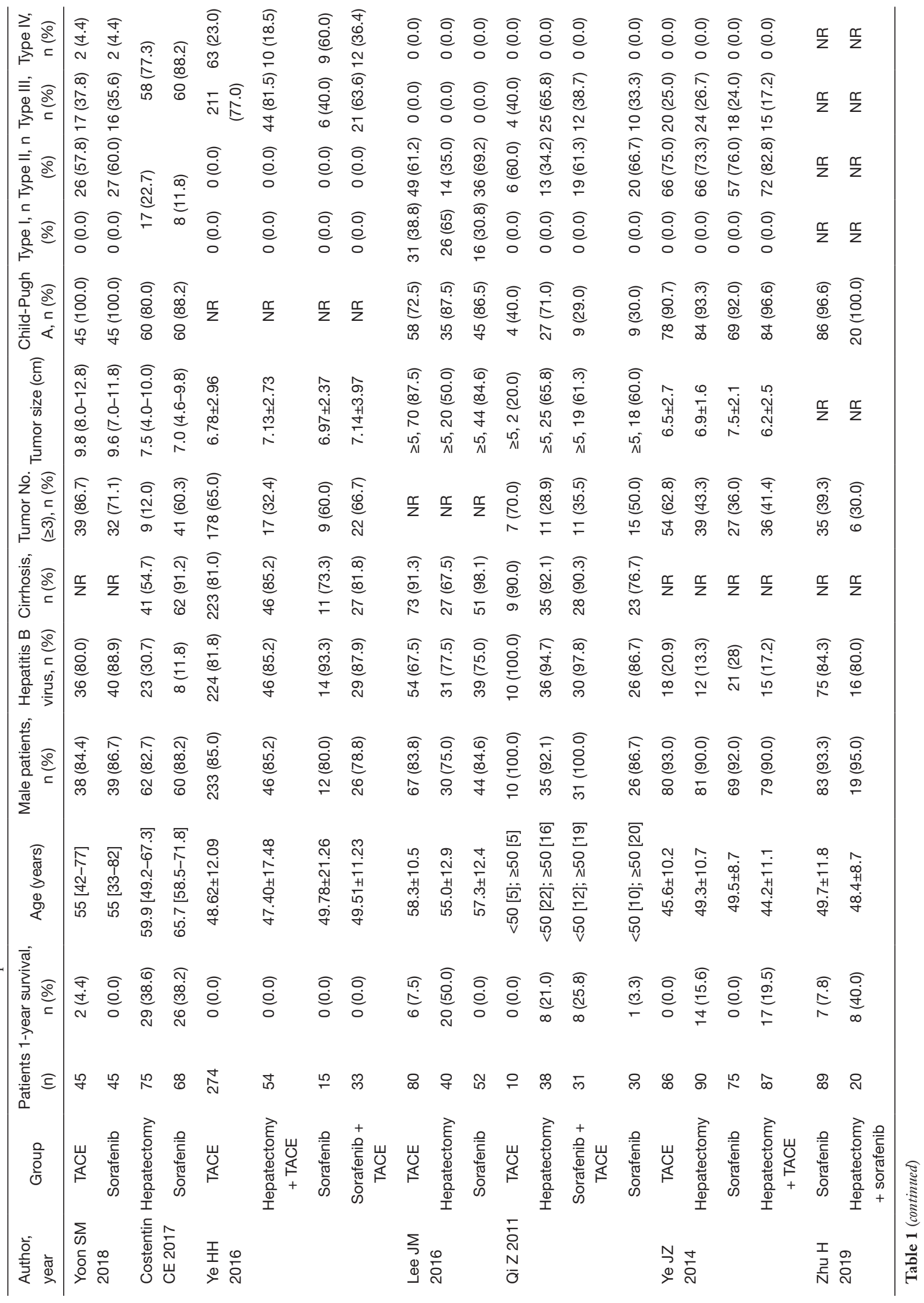




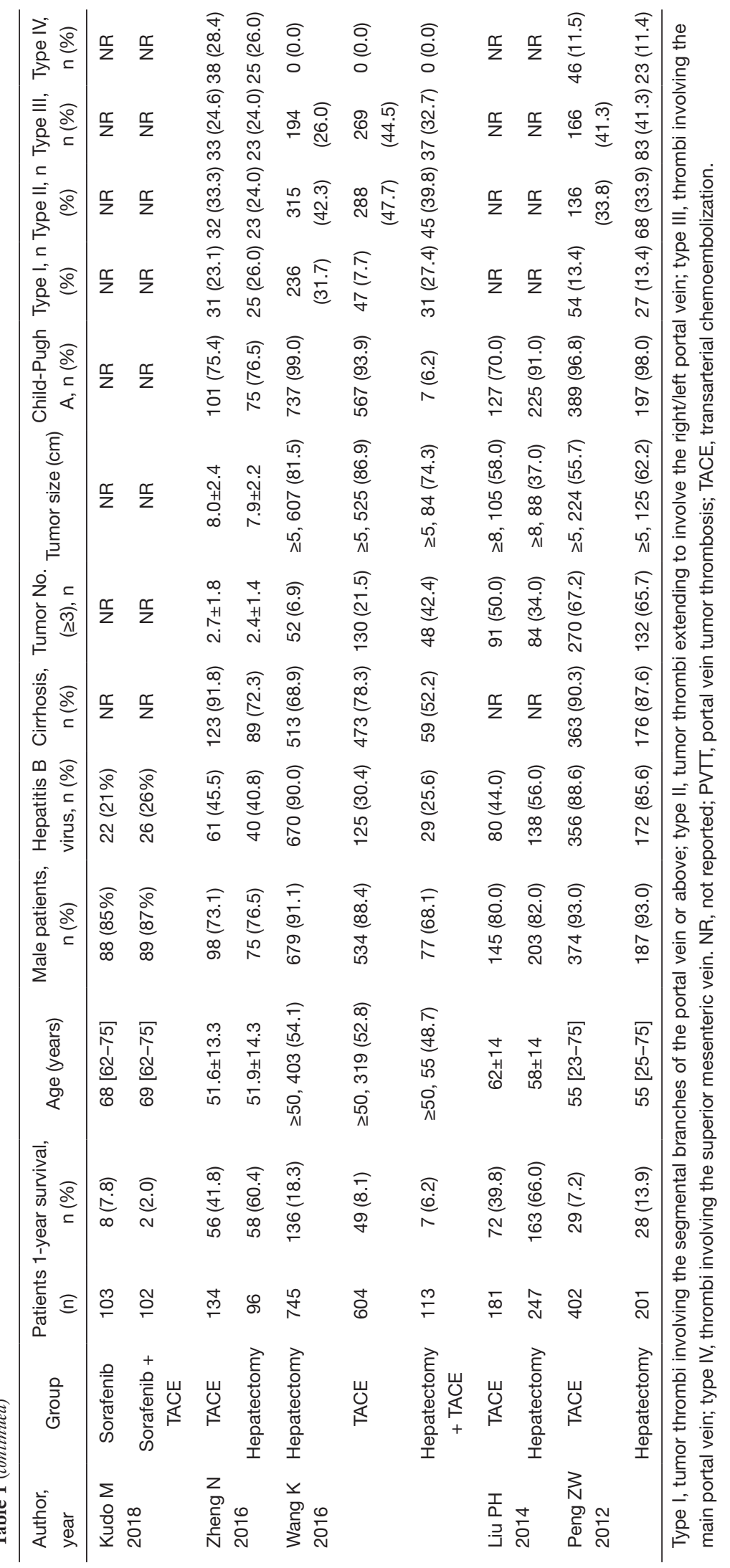




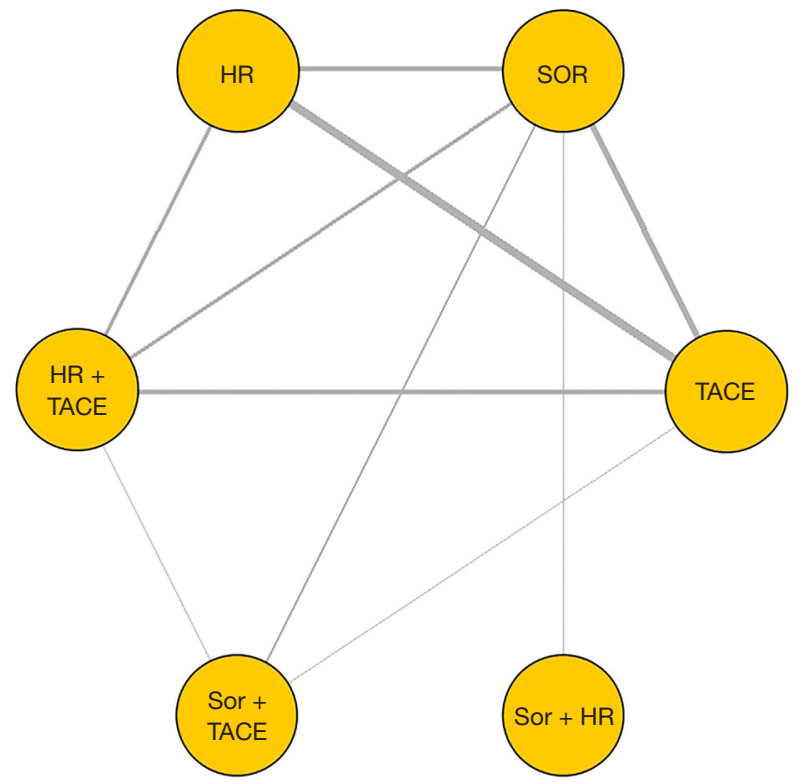

Figure 2 Network structure of different treatments compared to each other. HR, hepatectomy; Sor, sorafenib; TACE, transarterial chemoembolization

\section{Results of the Brooks-Gelman-Rubin diagnostic plot and potential scale reduction factor value}

The result of the Brooks-Gelman-Rubin diagnostic plot is shown in Figure 3. After 100,000 iterations, both the median and $97.5 \%$ value of the shrink factor tended to be 1 and gradually stabilized. The potential scale reduction factor (PFRF) value of each comparison was close to 1 after 100,000 iterations as well. That meant that the convergence could be confirmed accurately (35).

\section{Analysis of outcomes}

The network meta-analysis ORs and 95\% CIs for the effectiveness of different treatments are presented in Figure 4. The forest plots are presented Figure S2. All the OR intervals are not between " 0 " and " 1 ". This means that we can't use these results to extrapolate which treatment is most effective. We can infer that one of those treatments may be a better choice. As shown in Figure 5 and Table S1, the results of the rank probability of effectiveness showed that sorafenib + TACE was considered the most effective treatment, sorafenib + TACE group was ranged rank 1 when compared with the others (hepatectomy group: OR: 0.79, 95\% CI: 0.03-18.26; hepatectomy + TACE group:
OR: $0.51,95 \%$ CI: 0.01-13.59; sorafenib group: OR: 0.14, 95\% CI: 0.01-2.29; sorafenib + hepatectomy group: OR: 0.15, 95\% CI: 0.00-24.88; and TACE group: OR: 0.51; 95\% CI: 0.02-9.88). The second most effect treatment option was hepatectomy alone, hepatectomy group was ranged rank 2 when compared with the others (sorafenib + TACE group: OR: 1.32, 95\% CI: 0.05-39.64; hepatectomy + TACE group: OR: 0.71: 0.07-6.10; sorafenib group: OR: 0.18, 95\% CI: $0.02-1.17$, sorafenib + hepatectomy: OR: 0.19, 95\% CI: 0.00-22.48; and TACE group: OR: 0.65, 95\% CI: 0.13-3.28). Sorafenib or sorafenib + hepatectomy was considered the least effective treatment option.

\section{Discussion}

Since the Sorafenib Hepatocellular Carcinoma Assessment Randomized Protocol (SHARP) trial in 2007, sorafenib is the only recommended therapy when HCC is associated with PVTT, which can effectively prolong the survival of PVTT patients $(8,36-40)$. Considering the tolerance profile of this drug and the limited efficacy when used to treat PVTT patients (median overall survival rate of 8.1 months in SHARP key trials), the most effective management of HCC in PVTT patients is regularly questioned (40).

In some areas, sorafenib is expensive and not readily available. Surgical resection and TACE are also considered alternative treatments for patients with advanced liver cancer.

Based on the findings of the present study, the survival time of HCC patients with PVTT varies depending on treatment method. Hepatectomy could achieve longer survival times compared with sorafenib, while the effect of TACE on the survival of PVTT patients is not statistically significant compared with sorafenib (38).

In a recent study, advanced liver cancer patients receiving sorafenib required multimode therapy for optimal outcomes, including hepatectomy after sorafenib treatment, treatment of complications, TACE and other adjuvant treatments, and the rational use of sorafenib after surgery (41). Zhang et al. reported hepatectomy might be more effective in patients without PVTT in the main trunk than TACE or conservative treatment (16). While in the results of this study, hepatectomy alone is the second effective treatment option. Both two results considered that hepatectomy is an effective approach. We further considered "Sorafenib + TACE" as an independent group to compare with other groups.

Invasion of the portal vein or a tumor thrombus in the 
AI

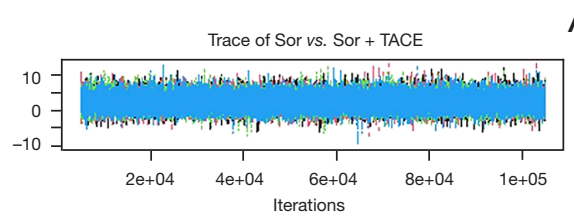

A2

A4

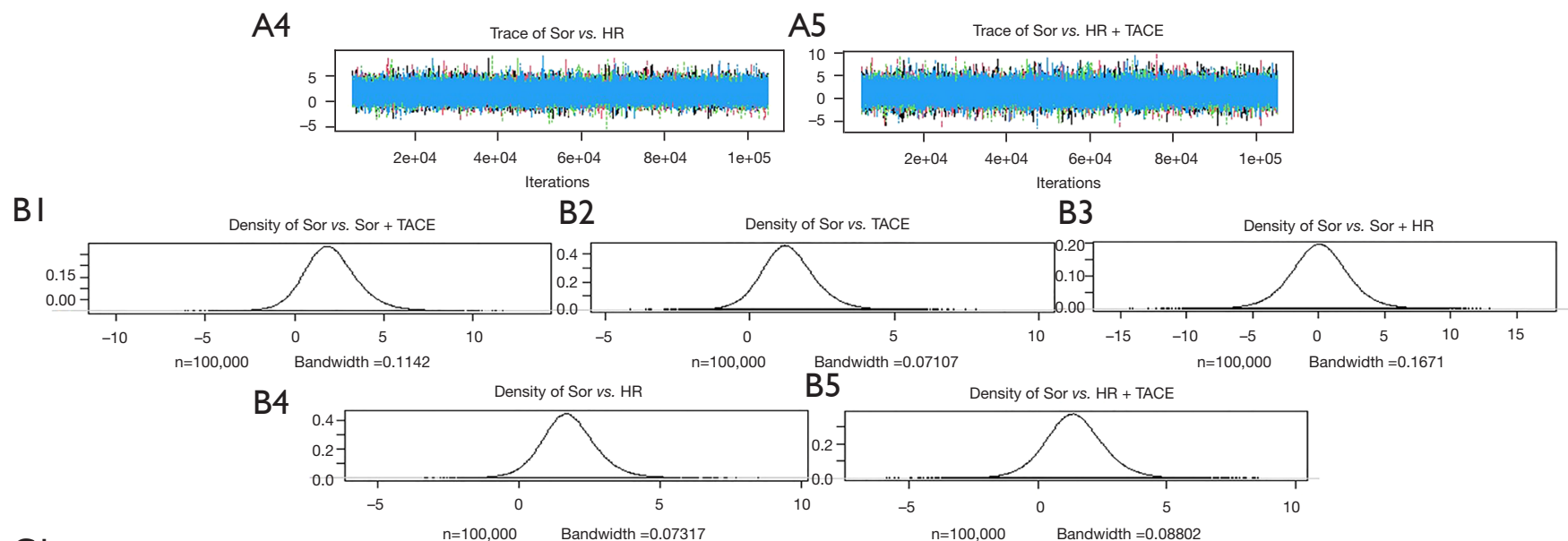

A3

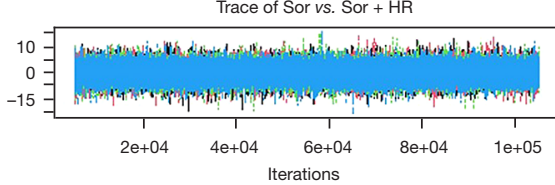

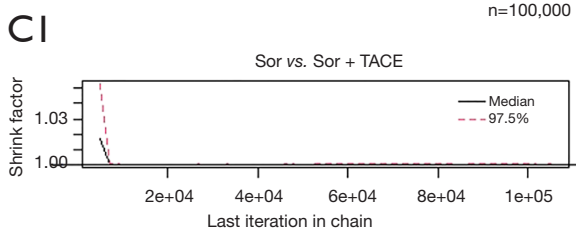

C2 Sor vs. TACE

C3

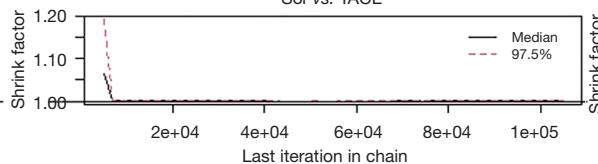

C4

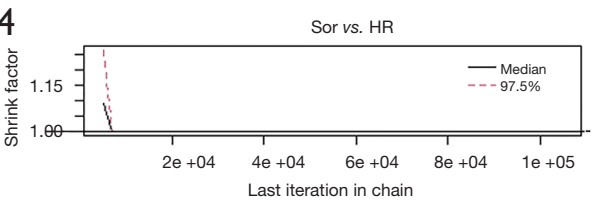

C5 Sor vs. HR + TACE
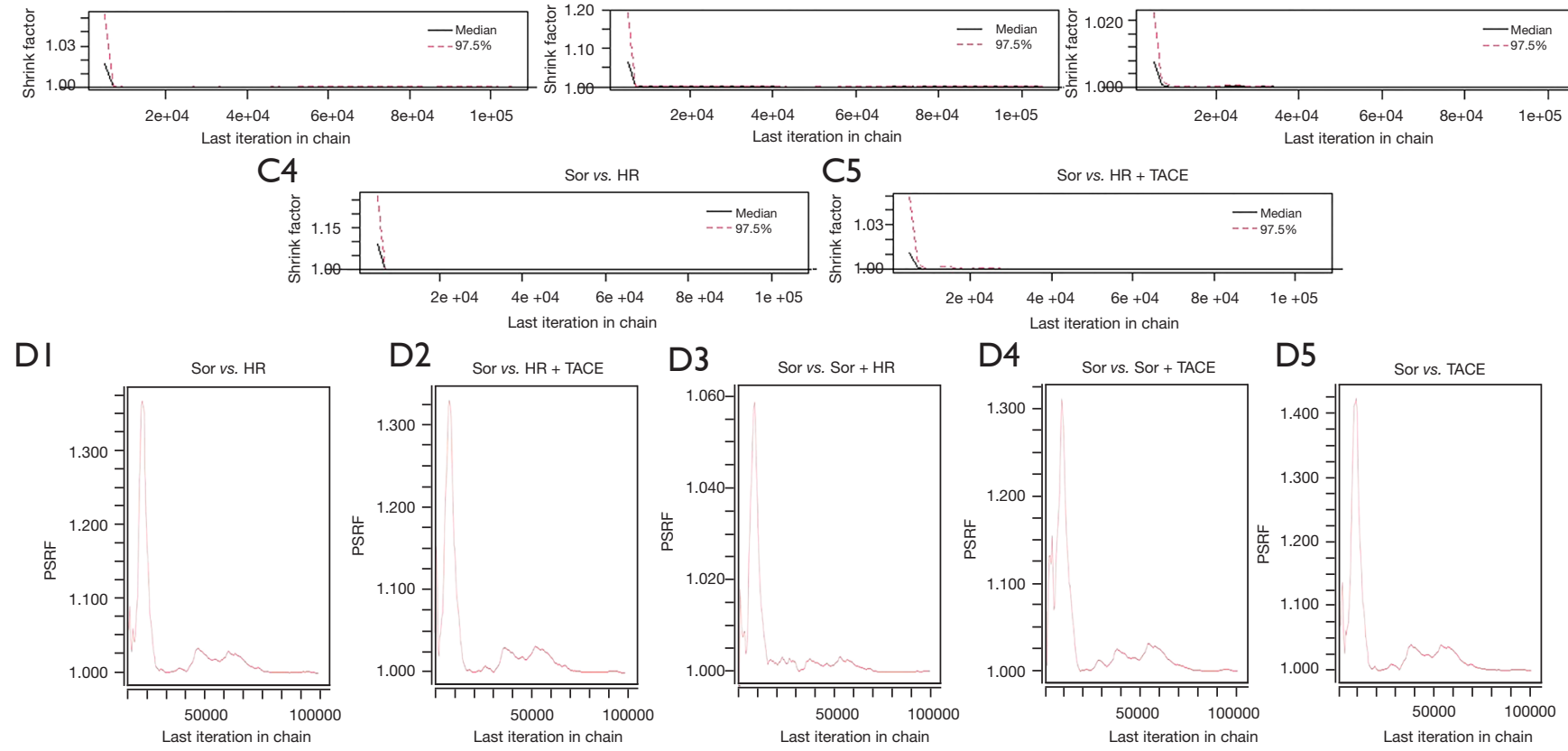

Figure 3 Result of convergence verification. HR, hepatectomy; PSRF, potential scale reduction factor; Sor, sorafenib; TACE, transarterial chemoembolization.

portal vein may promote intrahepatic metastasis, as well as decrease in blood supply and high portal pressure due to blockage of the blood vessels, eventually leading to deterioration of liver function with portal hypertension complications (42). It is for this reason that treatment options are limited. Because of the rapid progression of tumor thrombus, quick removal or reduction of tumor thrombus volume is important in the course of treatment. However, sorafenib is only likely to delay tumor progression, and the incidence of objective responses is low $(2-3 \%)$. TACE has limited efficacy in reducing tumor thrombus volume $(43,44)$. 


\begin{tabular}{|l|l|l|l|l|l|}
\hline HR & $0.71(0.07,6.10)$ & $0.18(0.02,1.17)$ & $0.19(0.00,22.48)$ & $1.32(0.05,39.64)$ & $0.65(0.13,3.28)$ \\
\hline $1.42(0.17,12.67)$ & HR + TACE & $0.25(0.02,2.48)$ & $0.28(0.00,37.57)$ & $1.84(0.07,68.71)$ & $0.91(0.12,7.98)$ \\
\hline $5.60(0.86,41.97)$ & $4.02(0.40,43.09)$ & Sor & $1.11(0.01,88.37)$ & $7.17(0.44,197.62)$ & $3.61(0.61,26.66)$ \\
\hline $5.311(0.05,793.39)$ & $3.82(0.03,656.24)$ & $0.95(0.01,92.79)$ & Sor + HR & $6.72(0.04,2138.35)$ & $3.49(0.03,508.55)$ \\
\hline $0.79(0.03,18.26)$ & $0.51(0.01,13.59)$ & $0.14(0.01,2.29)$ & $0.15(0.00,24.88)$ & Sor + TACE & $0.51(0.02,9.88)$ \\
\hline $1.54(0.30,7.16)$ & $1.11(0.13,8.29)$ & $0.29(0.04,1.63)$ & $0.27(0.00,32.99)$ & $2.01(0.10,54.56)$ & TACE \\
\hline
\end{tabular}

Figure 4 ORs and 95\% CIs for the effectiveness of different treatments. Results are the ORs in the column-defining treatment compared with the ORs in the row-defining treatment. For efficacy, ORs $>1$ favor the column-defining treatment. For acceptability, ORs $<1$ favor the first treatment in the order. HR, hepatectomy; Sor, sorafenib; TACE, transarterial chemoembolization; ORs, odds ratios; CIs, confidence intervals.

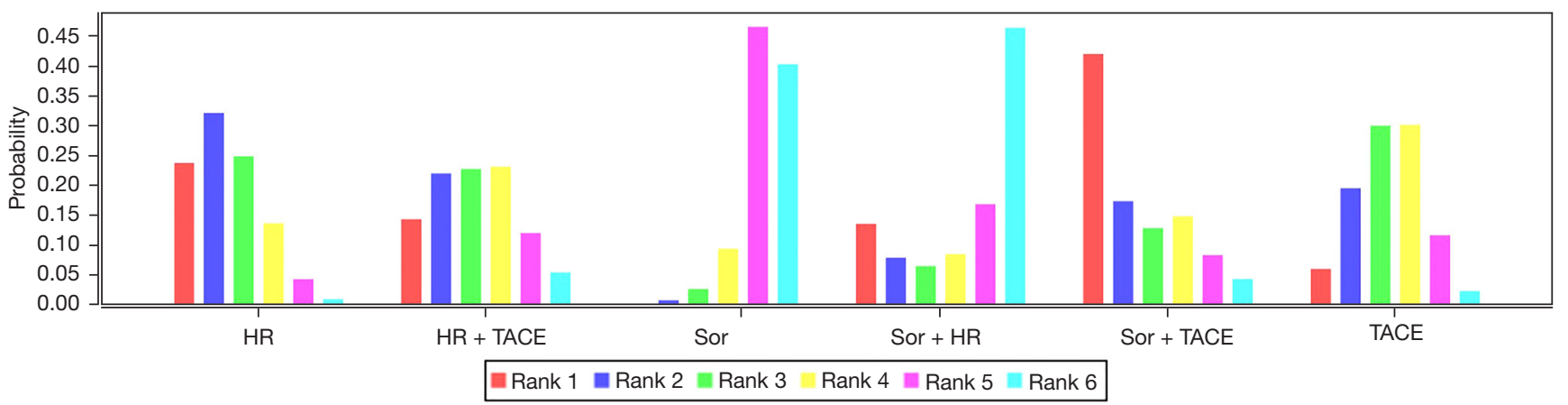

Figure 5 Rank probability of effectiveness. Rank 1 indicates most effective, rank 6 indicates least effective. HR, hepatectomy; Sor, sorafenib; TACE, transarterial chemoembolization.

The present study has some limitations. First, most studies included in this study were designed retrospectively, we need to include more prospective randomized clinical trials of high-quality to refine our study, further confirm and improve the accuracy of the results. Second, in most previously published studies, most patients have hepatitis B virus. Therefore, additional studies are needed for patients without hepatitis B virus infection. Third, the study of sorafenib has its own limitations, mainly in terms of whether the patients are following the doctor's advice and if they are being regularly followed up, a sensitivity analysis needed to be performed. Fourth, PVTT patients were not studied in groups according to the location of the tumor thrombus. Fifth, some articles did not provide tumor features; therefore, imbalance between the 6 groups of treatments may exist. Finally, the data were uneven distributed around the world, with more from Asia. Further studies will need to have a focus on Western data.

According to the results of Figure 4, it can be seen that all the OR intervals were not between 0 and 1 . This means that we can't use these results to extrapolate which treatment is most effective. We can only infer that one of those treatments may be the best choice. Such results may be related to insufficient sample size. It may also be related to the baseline inconsistency of the classification of the type of PVTT included in the article. More literature is needed to confirm this. Furthermore, according to Figure 3, it can be seen that the bandwidth value did not get closely to 0 . This result is possibly related to the lack of the comparison between the sorafenib group and sorafenib + hepatectomy group in the included literature. Finally, subgroup analysis needs to be further refined in future studies. Further data to refine the results of the present study are required.

The results of the rank probability of effectiveness showed sorafenib + TACE was the most effective treatment, sorafenib + TACE group was ranged rank 1 when compared with the others (hepatectomy group: OR: $0.79,95 \%$ CI: 0.03-18.26; hepatectomy + TACE group: OR: 0.51, 
95\% CI: 0.01-13.59; sorafenib group: OR: 0.14, $95 \%$ CI: 0.01-2.29; sorafenib + hepatectomy group: OR: 0.15, 95\% CI: 0.00-24.88; and TACE group: OR: 0.51, 95\% CI: 0.02-9.88). The second most effective treatment was hepatectomy alone. Our results suggest that for HCC patients with PVTT, combined therapy is better than single therapy. In patients without surgical conditions, TACE combined with Sorafenib may be more effective than TACE alone or sorafenib alone. For patients who still have conditions for surgery hepatectomy may be a useful option.

\section{Acknowledgments}

Thanks to West China Hospital of Sichuan University and the Department of General Surgery for its support. Thanks for the technical support of Jinbang Kou.

Funding: This study was supported by the Key Technology Research and Development Program of the Sichuan Province (No. 2017FZ0082 and No. 2019YFS0208), National Natural Science Foundation of China grants (No. 71673193), Chinese Foundation for Hepatitis Prevention and Control-Tian Qing Liver Disease Research Fund Subject (No. TQGB20190202).

\section{Footnote}

Reporting Checklist: The authors have completed the PRISMA reporting checklist. Available at https://dx.doi. org/10.21037/atm-21-3937

Conflicts of Interest: All authors have completed the ICMJE uniform disclosure form (available at https://dx.doi. org/10.21037/atm-21-3937). The authors have no conflicts of interest to declare.

Ethics Statement: The authors are accountable for all aspects of the work in ensuring that questions related to the accuracy or integrity of any part of the work are appropriately investigated and resolved. This study was approved by Liver Surgery and Liver Transplantation Center, West China Hospital of Sichuan University.

Open Access Statement: This is an Open Access article distributed in accordance with the Creative Commons Attribution-NonCommercial-NoDerivs 4.0 International License (CC BY-NC-ND 4.0), which permits the noncommercial replication and distribution of the article with the strict proviso that no changes or edits are made and the original work is properly cited (including links to both the formal publication through the relevant DOI and the license). See: https://creativecommons.org/licenses/by-nc-nd/4.0/.

\section{References}

1. Torre LA, Bray F, Siegel RL, et al. Global cancer statistics, 2012. CA Cancer J Clin 2015;65:87-108.

2. Ando E, Tanaka M, Yamashita F, et al. Hepatic arterial infusion chemotherapy for advanced hepatocellular carcinoma with portal vein tumor thrombosis: analysis of 48 cases. Cancer 2002;95:588-95.

3. Poon RT, Fan ST, Lo CM, et al. Intrahepatic recurrence after curative resection of hepatocellular carcinoma: longterm results of treatment and prognostic factors. Ann Surg 1999;229:216-22.

4. Kokudo T, Hasegawa K, Matsuyama Y, et al. Survival benefit of liver resection for hepatocellular carcinoma associated with portal vein invasion. J Hepatol 2016;65:938-43.

5. Lin DX, Zhang QY, Li X, et al. An aggressive approach leads to improved survival in hepatocellular carcinoma patients with portal vein tumor thrombus. J Cancer Res Clin Oncol 2011;137:139-49.

6. Llovet JM, Bustamante J, Castells A, et al. Natural history of untreated nonsurgical hepatocellular carcinoma: rationale for the design and evaluation of therapeutic trials. Hepatology 1999;29:62-7.

7. Takizawa D, Kakizaki S, Sohara N, et al. Hepatocellular carcinoma with portal vein tumor thrombosis: clinical characteristics, prognosis, and patient survival analysis. Dig Dis Sci 2007;52:3290-5.

8. Llovet JM, Ricci S, Mazzaferro V, et al. Sorafenib in advanced hepatocellular carcinoma. N Engl J Med 2008;359:378-90.

9. Kenjo A, Miyata H, Gotoh M, et al. Risk stratification of 7,732 hepatectomy cases in 2011 from the National Clinical Database for Japan. J Am Coll Surg 2014;218:412-22.

10. Chen XP, Qiu FZ, Wu ZD, et al. Effects of location and extension of portal vein tumor thrombus on longterm outcomes of surgical treatment for hepatocellular carcinoma. Ann Surg Oncol 2006;13:940-6.

11. Chen ZH, Zhang XP, Wang K, et al. Liver resection versus transcatheter arterial chemoembolization for the treatment of patients with hepatocellular carcinoma and hepatic vein or inferior vena cava tumor thrombus: A propensity score matching analysis. Hepatol Res 2019;49:441-52. 
12. Lee JM, Jang BK, Lee YJ, et al. Survival outcomes of hepatic resection compared with transarterial chemoembolization or sorafenib for hepatocellular carcinoma with portal vein tumor thrombosis. Clin Mol Hepatol 2016;22:160-7.

13. Yamada R, Kishi K, Sato M, et al. Transcatheter arterial chemoembolization (TACE) in the treatment of unresectable liver cancer. World J Surg 1995;19:795-800.

14. Schwarz RE, Abou-Alfa GK, Geschwind JF, et al. Nonoperative therapies for combined modality treatment of hepatocellular cancer: expert consensus statement. HPB (Oxford) 2010;12:313-20.

15. Zhang ZM, Lai EC, Zhang C, et al. The strategies for treating primary hepatocellular carcinoma with portal vein tumor thrombus. Int J Surg 2015;20:8-16.

16. Zhang ZY, Dong KS, Zhang EL, et al. Resection might be a meaningful choice for hepatocellular carcinoma with portal vein thrombosis: A systematic review and metaanalysis. Medicine (Baltimore) 2019;98:e18362.

17. Liang L, Chen TH, Li C, et al. A systematic review comparing outcomes of surgical resection and nonsurgical treatments for patients with hepatocellular carcinoma and portal vein tumor thrombus. HPB (Oxford) 2018;20:1119-29.

18. Rabelo CC, Feres M, Gonçalves C, et al. Systemic antibiotics in the treatment of aggressive periodontitis. A systematic review and a Bayesian Network meta-analysis. J Clin Periodontol 2015;42:647-57.

19. Cirillo M, Venturini M, Ciccarelli L, et al. Clinician versus nurse symptom reporting using the National Cancer Institute-Common Terminology Criteria for Adverse Events during chemotherapy: results of a comparison based on patient's self-reported questionnaire. Ann Oncol 2009;20:1929-35.

20. Shi J, Lai EC, Li N, et al. Surgical treatment of hepatocellular carcinoma with portal vein tumor thrombus. Ann Surg Oncol 2010;17:2073-80.

21. Zhou Q, Wang Y, Zhou X, et al. Prognostic analysis for treatment modalities in hepatocellular carcinomas with portal vein tumor thrombi. Asian Pac J Cancer Prev 2011;12:2847-50.

22. Ye JZ, Zhang YQ, Ye HH, et al. Appropriate treatment strategies improve survival of hepatocellular carcinoma patients with portal vein tumor thrombus. World J Gastroenterol 2014;20:17141-7.

23. Ye HH, Ye JZ, Xie ZB, et al. Comprehensive treatments for hepatocellular carcinoma with tumor thrombus in major portal vein. World J Gastroenterol 2016;22:3632-43.
24. Yoon SM, Ryoo BY, Lee SJ, et al. Efficacy and Safety of Transarterial Chemoembolization Plus External Beam Radiotherapy vs Sorafenib in Hepatocellular Carcinoma With Macroscopic Vascular Invasion: A Randomized Clinical Trial. JAMA Oncol 2018;4:661-9.

25. Zhu H, Ye B, Qiao Z, et al. Hepatectomy combined with sorafenib in patients with intermediate-advanced hepatocellullar carcinoma. J BUON 2019;24:1382-9.

26. Zheng N, Wei X, Zhang D, et al. Hepatic resection or transarterial chemoembolization for hepatocellular carcinoma with portal vein tumor thrombus. Medicine (Baltimore) 2016;95:e3959.

27. Peng ZW, Guo RP, Zhang YJ, et al. Hepatic resection versus transcatheter arterial chemoembolization for the treatment of hepatocellular carcinoma with portal vein tumor thrombus. Cancer 2012;118:4725-36.

28. Wang K, Guo WX, Chen MS, et al. Multimodality Treatment for Hepatocellular Carcinoma With Portal Vein Tumor Thrombus: A Large-Scale, Multicenter, Propensity Mathching Score Analysis. Medicine (Baltimore) 2016;95:e3015.

29. Kudo M, Ueshima K, Yokosuka O, et al. Sorafenib plus low-dose cisplatin and fluorouracil hepatic arterial infusion chemotherapy versus sorafenib alone in patients with advanced hepatocellular carcinoma (SILIUS): a randomised, open label, phase 3 trial. Lancet Gastroenterol Hepatol 2018;3:424-32.

30. Costentin CE, Decaens T, Laurent A, et al. Sorafenib vs surgical resection for hepatocellular carcinoma with macrovascular invasion: A propensity score analysis. Liver Int 2017;37:1869-76.

31. Liu PH, Lee YH, Hsia CY, et al. Surgical resection versus transarterial chemoembolization for hepatocellular carcinoma with portal vein tumor thrombosis: a propensity score analysis. Ann Surg Oncol 2014;21:1825-33.

32. Toft N, Innocent GT, Gettinby G, et al. Assessing the convergence of Markov Chain Monte Carlo methods: an example from evaluation of diagnostic tests in absence of a gold standard. Prev Vet Med 2007;79:244-56.

33. van Valkenhoef G, Lu G, de Brock B, et al. Automating network meta-analysis. Res Synth Methods 2012;3:285-99.

34. Brooks SP, Gelman A. General Methods for Monitoring Convergence of Iterative Simulations. Journal of Computational and Graphical Statistics 1998;7:434-55.

35. Nelson T, Song JJ, Chin YM, et al. Bayesian Correction for Misclassification in Multilevel Count Data Models. Comput Math Methods Med 2018;2018:3212351.

36. Okuda K, Ohtsuki T, Obata H, et al. Natural history of 
hepatocellular carcinoma and prognosis in relation to treatment. Study of 850 patients. Cancer 1985;56:918-28.

37. European Association For The Study Of The Liver; European Organisation For Research And Treatment Of Cancer. EASL-EORTC clinical practice guidelines: management of hepatocellular carcinoma. J Hepatol 2012;56:908-43. Erratum in: J Hepatol 2012;56:1430.

38. Heimbach JK, Kulik LM, Finn RS, et al. AASLD guidelines for the treatment of hepatocellular carcinoma. Hepatology 2018;67:358-80.

39. Cheng AL, Kang YK, Chen Z, et al. Efficacy and safety of sorafenib in patients in the Asia-Pacific region with advanced hepatocellular carcinoma: a phase III randomised, double-blind, placebo-controlled trial. Lancet Oncol 2009;10:25-34.

40. Bruix J, Raoul JL, Sherman M, et al. Efficacy and safety of sorafenib in patients with advanced hepatocellular carcinoma: subanalyses of a phase III trial. J Hepatol 2012;57:821-9.

Cite this article as: Luo J, Xu L, Li L, Zhang J, Zhang M, $\mathrm{Xu}$ M. Comparison of treatments for hepatocellular carcinoma patients with portal vein thrombosis: a systematic review and network meta-analysis. Ann Transl Med 2021;9(18):1450. doi: 10.21037/atm-21-3937
41. Tanaka K, Shimada M, Kudo M. Characteristics of longterm survivors following sorafenib treatment for advanced hepatocellular carcinoma: report of a workshop at the 50th Annual Meeting of the Liver Cancer Study Group of Japan. Oncology 2014;87 Suppl 1:104-9.

42. Yoon SM, Lim YS, Won HJ, et al. Radiotherapy plus transarterial chemoembolization for hepatocellular carcinoma invading the portal vein: long-term patient outcomes. Int J Radiat Oncol Biol Phys 2012;82:2004-11.

43. Koo JE, Kim JH, Lim YS, et al. Combination of transarterial chemoembolization and three-dimensional conformal radiotherapy for hepatocellular carcinoma with inferior vena cava tumor thrombus. Int J Radiat Oncol Biol Phys 2010;78:180-7.

44. Kim GA, Shim JH, Yoon SM, et al. Comparison of chemoembolization with and without radiation therapy and sorafenib for advanced hepatocellular carcinoma with portal vein tumor thrombosis: a propensity score analysis. J Vasc Interv Radiol 2015;26:320-9.e6. 


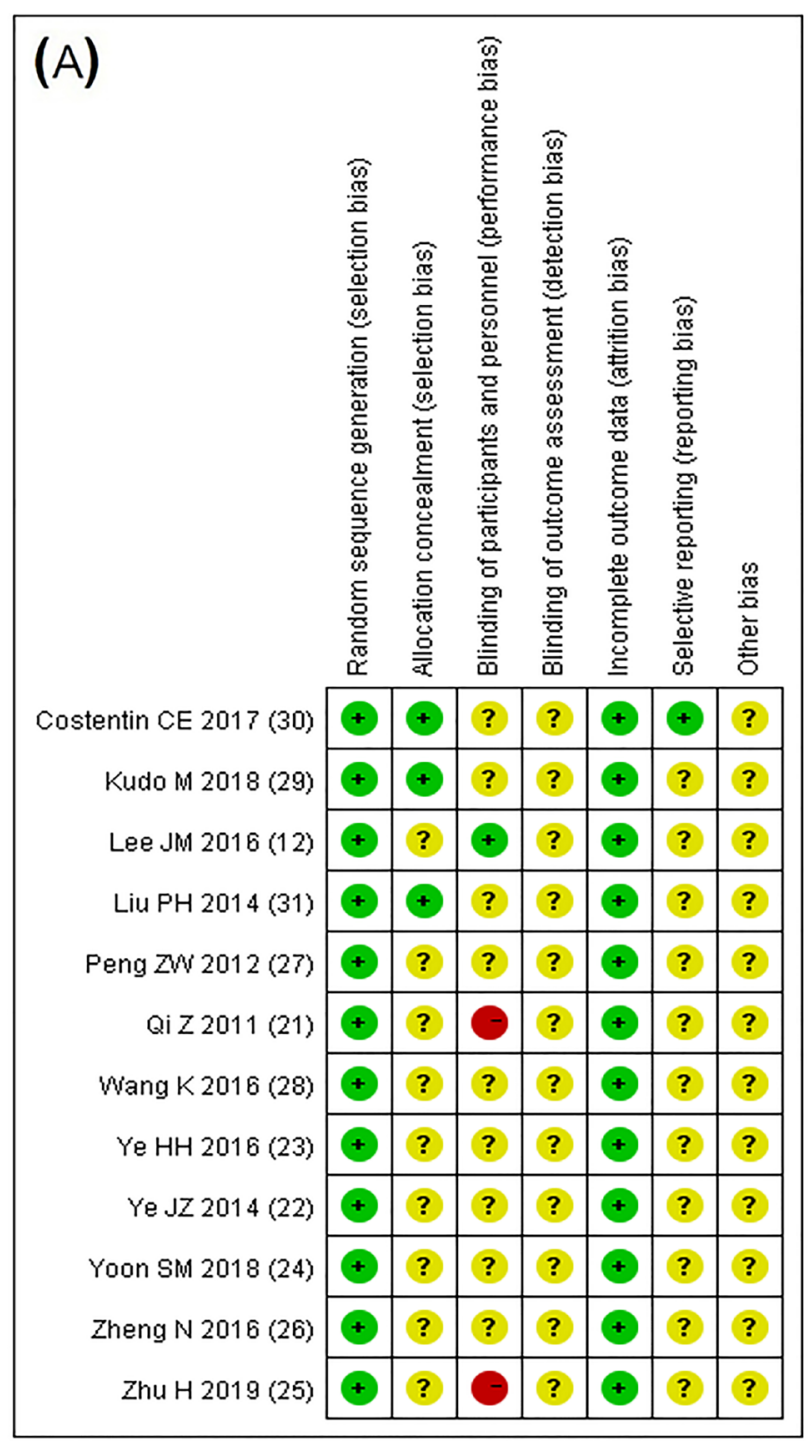

(B) Random sequence generation (selection bias) Allocation concealment (selection bias)

Blinding of participants and personnel (performance bias) Blinding of outcome assessment (detection bias) Incomplete outcome data (attrition bias) Selective reporting (reporting bias) Other bias

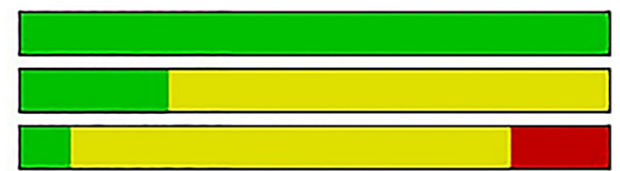

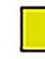

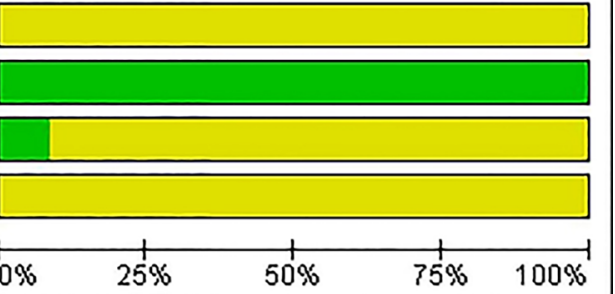

Low risk of bias $\quad \square$ Unclear risk of bias

High risk of bias

Figure S1 Risk of bias in the eligible studies. (A) Risk of bias summary. (B) Risk of bias graph. 
(A)

Compared with Sor + TACE

TACE

Sor

HR + TACE

SOr + HR

(D)

Compared with Sor + HR

TACE

Sor

$H R+$ TACE

Sor + TACE

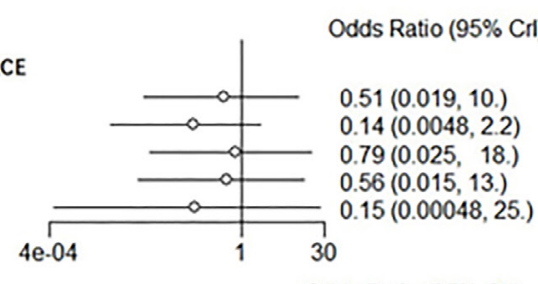

Odds Ratio $(95 \% \mathrm{Cr})$

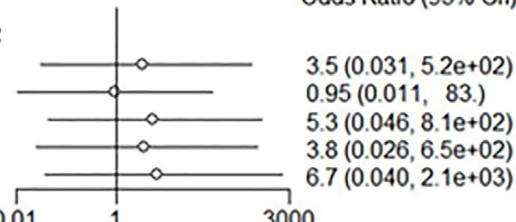

$0.95(0.011,83$.

$8(0.026,6.5 \mathrm{e}+02)$
(B)

Compared with $H R+$ TACE

TACE

Sor
HR

Sor + TACE

SOr + TACE
Sor + HR

(E)

Compared with $\mathrm{HR}$

TACE

Sor
HR + TACE

Sor + TACE

Sor $+H R$

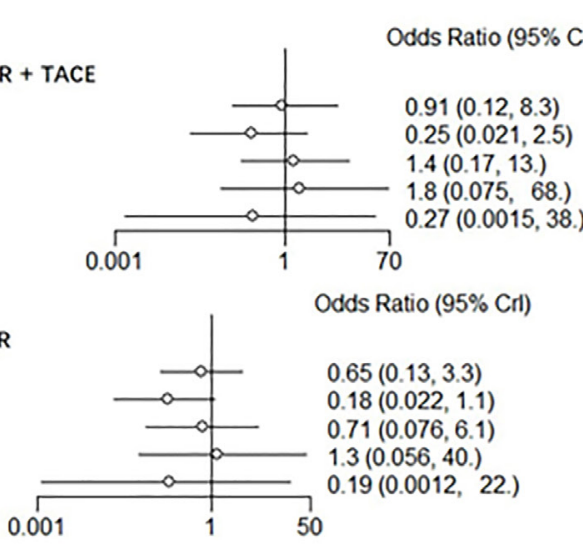

(C)

Compared with TACE

Sor

HR

HR + TACE

Sor + TACE

(F)

Compared with Sor

TACE

HR
HR + TACE

Sor + TACE

Sor + HR

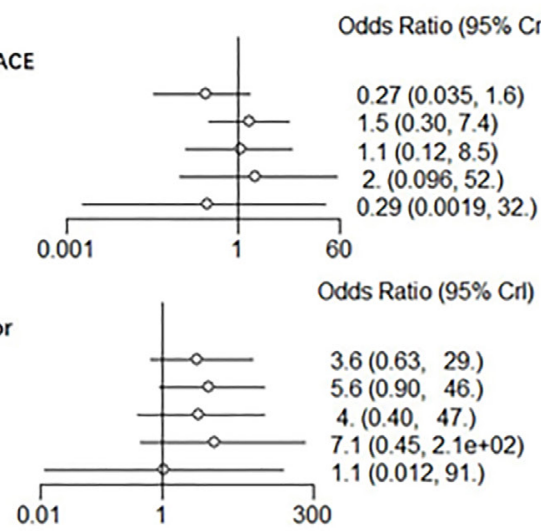

Figure S2 Forest plot results. HR, hepatectomy; Sor, sorafenib; TACE, transarterial chemoembolization.

Table S1 Rank probability of effectiveness

\begin{tabular}{lllllll}
\hline Treatment & Rank 1 (\%) & Rank 2 (\%) & Rank 3 (\%) & Rank 4 (\%) & Rank 5 (\%) & Rank 6 (\%) \\
\hline HR & 0.23 & 0.32 & 0.26 & 0.14 & 0.05 & 0.01 \\
HR+TACE & 0.14 & 0.22 & 0.23 & 0.24 & 0.13 & 0.05 \\
Sor & 0 & 0.01 & 0.03 & 0.1 & 0.46 & 0.41 \\
Sor + HR & 0.13 & 0.09 & 0.06 & 0.08 & 0.17 & 0.46 \\
Sor + TACE & 0.44 & 0.18 & 0.12 & 0.14 & 0.08 & 0.04 \\
TACE & 0.06 & 0.19 & 0.3 & 0.3 & 0.12 & 0.03 \\
\hline
\end{tabular}

Rank 1 indicates most effective, rank 6 indicates least effective. HR, hepatectomy; Sor, sorafenib; TACE, transarterial chemoembolization. 